\section{Pseudoaneurysm of the splenic artery mimicking a solid lesion}

\author{
Raffaele Pezzilli,' Monica Cevenini,' \\ Cristina Mosconi, ${ }^{2}$ Nico Pagano, ${ }^{1}$ \\ Matteo Renzulli, ${ }^{2}$ Rita Golfieri ${ }^{2}$
}

'Pancreas Unit, Department of Digestive System; ' Radiology Unit, Department of Diagnostic and Preventive Medicine, Sant'Orsola-Malpighi University Hospital, Bologna, Italy

\section{Abstract}

A 64-year-old man presented to the hospital because of hematemesis; on admission, he had weakness and pale skin, tachycardia and hypotension. Laboratory tests revealed severe anemia (hemoglobin $7.8 \mathrm{~g} / \mathrm{dL}$ ); liver, renal and pancreatic function tests were normal. An upper digestive endoscopy revealed a gastric ulcer of the cardia, treated with metallic clips and adrenalin injection. The patient was treated with fluids and was transfused with three units of red blood cells. In the previous two months, due to the presence of bloating and diarrhea, associated with abdominal distension, a colon-computed tomography (CT) revealed a large retroperitoneal hypodense mass, $53 \times 37 \mathrm{~mm}$ in size, without contrast enhancement localized between the body and the tail of the pancreas and the stomach, near the splenic artery and without signs of infiltration. To better define the mass, endoscopic ultrasound and biopsy were performed; however histopathology of multiple biopsies was not diagnostic, because of the presence of necrotic tissue and inflammatory cells. Since hematemesis recurred, the patient underwent a second upper digestive endoscopic examination, but no source of bleeding was found. Then a new contrast enhanced CT was performed that showed a size reduction of the mass, the presence of blood in the stomach and a small pseudoaneurysm of the splenic artery. Because of these findings an angiograpghic study was carried out; angiography confirmed a splenic artery pseudoaneurysm that was successfully embolized with metal microcoils.

\section{Introduction}

Splenic artery pseudoaneurysms are rare and difficult to recognize especially as in the present case in which the imaging feature of splenic aneurysm presented at our observation as solid abdominal mass.

\section{Case Report}

A 64-year-old man was admitted to our Department of Digestive System because of hematemesis. The patient had a previous history of multiple coronary stents for cardiac ischemia and he chronically assumed aspirin and clopidogrel. On admission, the patient had weakness and physical examination, he revealed pale skin, tachycardia (110 beats per minutes) and hypotension (arterial blood pressure: $80 / 50 \mathrm{mmHg}$ ). The physical examination was unremarkable and no pulsatile abdominal mass was detected. Laboratory tests revealed severe anemia, hemoglobin being $7.8 \mathrm{~g} / \mathrm{dL}$; liver, renal and pancreatic function tests were normal. An upper digestive endoscopy revealed a gastric ulcer of the cardia, treated with metallic clips and adrenalin injection. The patient was treated with fluids and was transfused with three units of red blood cells.

In the previous two months, due to the presence of bloating and diarrhea, associated with abdominal distension, a colon-computed tomography (CT) revealed diffused colonic uncomplicated diverticula; in addition, a large retroperitoneal hypodense mass, $53 \times 37 \mathrm{~mm}$ in size, without contrast enhancement was incidentally discovered, localized between the body and the tail of the pancreas and the stomach, near the splenic artery and without signs of infiltration (Figure 1). To better define the mass, endoscopic ultrasound was performed and a biopsy of the mass was carried out (Figure 2), but histopathology of multiple biopsies was not diagnostic, because of the presence of necrotic tissue and inflammatory cells.

The abdominal mass appeared to be not related to a pancreatic mass and seems to be contiguous to the stomach, the differential diagnosis was made between a gastric lesion such or lymphoma of the stomach because the presence of necrotic tissue within the lesion.

Since hematemesis recurred, the patient underwent a second upper digestive endoscopic examination, but no source of bleeding was found. Thus, a new triphasic multidetector CT (including an unenhanced and arterial, portal and venous phases) was performed. This latter examination showed a size reduction of the mass, the presence of blood in the stomach and a small (about $1.5 \mathrm{~cm}$ ) pseudoaneurysm of the splenic artery (Figure 3 ). Because of these findings an angiographic study was carried out. As a last examination, an angiography was carried out in order to evaluate and possibly treat the cause of gastrointestinal bleeding. Angiography confirmed a splenic artery pseudoaneurysm (Figure 4), subsequently embolized with metal microcoils (Figure 5) using coil isolation/packing technique. ${ }^{1}$
Correspondence: Raffaele Pezzilli, Pancreas Unit, Department of Digestive System, Sant'OrsolaMalpighi University Hospital, Bologna, Italy.

Tel./Fax: + 39.051.2144148.

E-mail: raffaele.pezzilli@aosp.bo.it

Key words: Abdominal mass; Pseudoaneurysm; Radiology; Endoscopic ultrasound.

Conflict of interest: the authors declare no potential conflict of interest.

Contributions: RP wrote the manuscript; RP, MC and NP followed the patient; CM, MR, RG performed the imaging and the interventional radiological approach; MC, CM and NP critically revised the manuscript. All authors approved the final version of the manuscript.

Note: written informed consent was obtained from the patient for publication of this case report, including accompanying images.

Received for publication: 11 March 2016.

Revision received: 22 June 2016.

Accepted for publication: 24 June 2016.

This work is licensed under a Creative Commons Attribution 4.0 License (by-nc 4.0).

(C) Copyright R. Pezzilli et al., 2016

Licensee PAGEPress, Italy

Emergency Care Journal 2016; 12:5880

doi:10.4081/ecj.2016.5880

\section{Discussion}

Splenic artery pseudoaneurysm incidence ranges from 0.01 to $0.2 \%$ of patients and increases up to $10 \%$ in older patients. ${ }^{2}$ Bleeding and/or abdominal pain/distension are the most common clinical manifestations of pseudoaneurysms, which are usually associated with pancreatitis, trauma or rarely abdominal diseases such as complicated peptic ulcer; 3 in these cases, gastrointestinal bleeding can be overt or also can extend into the peritoneum, retroperitoneal space, adjacent organs, or even into a closed space called pseudocyst. So far, in clinical practice no reported cases of unprovoked splenic artery pseudoaneurysm, ${ }^{3}$ have been reported as in the present patient. Hemodynamic instability is often present and this condition represents a true medical emergency. ${ }^{2}$ Once several other disorders as evident bleeding, severe hemolytic anemia, compensatory volume fluid shifts, sepsis, and large hematomas are excluded, large hematocrit declines that cannot be readily explained or unable to be visualized with standard endoscopic procedures must prompt the physician to the diagnostic approach of splenic artery pseudoaneurysm and its hemodynamic stabilization. The standard treatment 


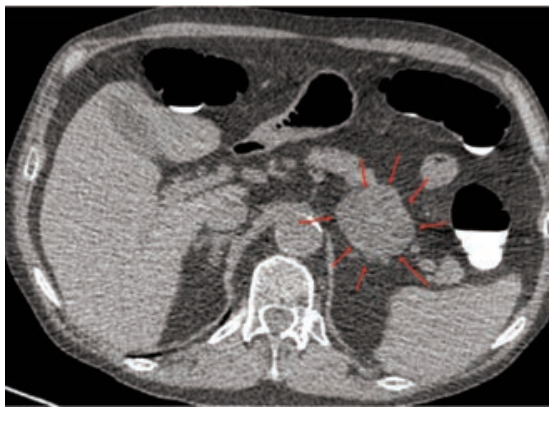

Figure 1. Computed tomography of the colon. Incidentally discovered large retroperitoneal hypodense mass, $53 \times 37$ $\mathrm{mm}$ in size localized between the body and the tail of the pancreas and the stomach (arrows).

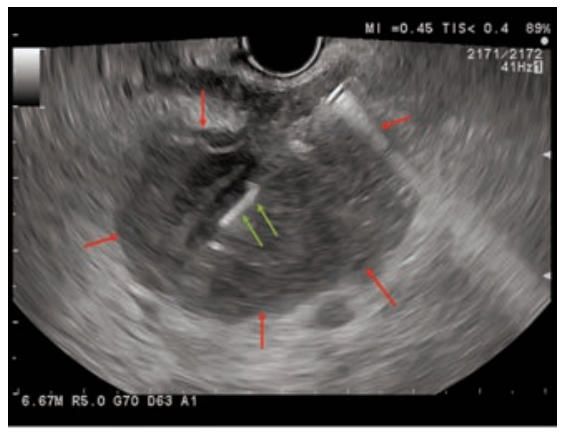

Figure 2. Endoscopic ultrasound and biopsy were performed to better define the mass (red arrows). The needle for the biopsy is indicated with green arrows.
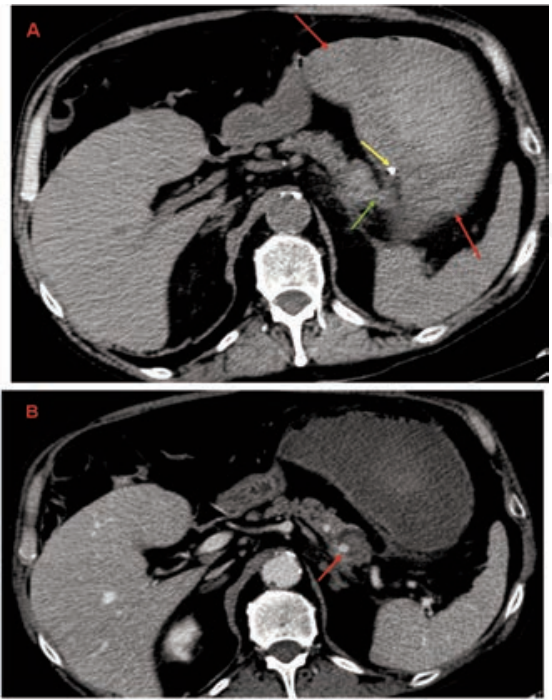

Figure 3. A) The unenhanced abdominal computed tomography showing the blood in the stomach (red arrows), the reduction in size of the mass (yellow arrow) and the presence of communication between the stomach and the lesion (green arrow). B) Contrast enhanced abdominal computed tomography shows the presence of arterial splenic pseudoaneurysm. for splenic artery pseudoaneurysm is angiographic-directed embolization, with either coils, or, less frequently, stent. ${ }^{4-6}$

\section{Conclusions}

The treatment of choice of splenic artery pseudoaneurysm is operator-dependent based on the tortuosity of the target vessel, the location, morphology and size of the lesion. Stent approach has the advantage of excluding the aneurysm from systemic blood flow without impeding the flow of blood to the spleen; however, as described in the literature, it is limited by rigid delivery systems which can preclude stent placement in distal tortuous branches, as commonly seen in splenic vasculature. ${ }^{7}$ In this case, the tortuosity of the vessel did not allow us to use stent; moreover, it is not always easy to advance a large bore sheath within the tortuous splenic artery. Solid pseudoaneurism is a very rare finding in clinical practice; the presence of hypodense mass, without contrast

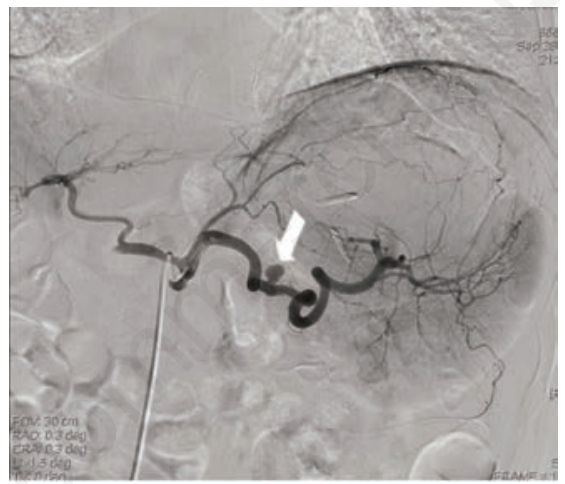

Figure 4. The angiographic study confirmed a splenic artery pseudoaneurysm (arrow).

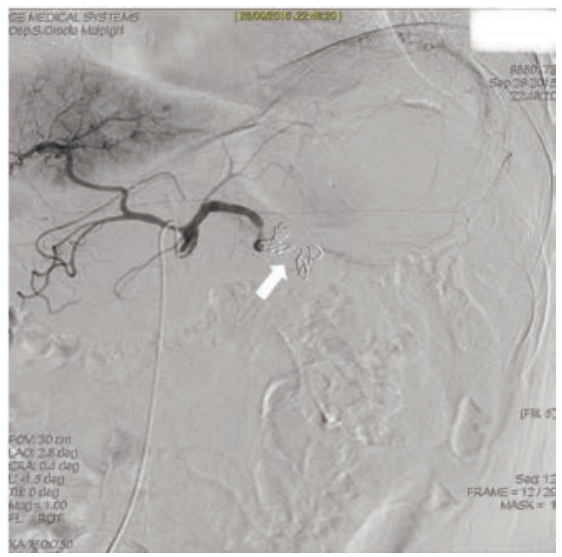

Figure 5. Pseudoaneurysm isolated by isolation/packing technique (arrow). enhancement and the presence of necrotic tissue and inflammatory cells on histological specimen may lead to an angiographic study in order to confirm the origin of the lesion.

\section{References}

1. Yamamoto S, Hirota S, Maeda $\mathrm{H}$, et al. Transcatheter coil embolization of splenic artery aneurysm. Cardiovasc Intervent Radiol 2008;31:527-34.

2. Barakat B, Pezzilli R. Imaging sketch of bedside ultrasonography to improve the diagnosis of rupture of splenic artery aneurysm. Emerg Care J 2015;11:5257.

3. Schatz RA, Schabel S, Rockey DC. Idiopathic splenic artery pseudoaneurysm rupture as an uncommon cause of hemorrhagic shock. J Investig Med High Impact Case Rep 2015;3:2324709615577816.

4. Arepally A, Dagli M, Hofmann LV, et al. Treatment of splenic artery aneurysm with the use of stent-graft. J Vasc Interv Radiol 2002;13:631-3.

5. Kulkarni CB, Moorthy S, Pullara SK, Kannan RR. Endovascular treatment of aneurysm of splenic artery arising from splenomesentric trunk using stent graft. Korean J Radiol 2013;14:931-4.

6. Kulkarni CB, Moorthy S, Pullara SK, et al. Endovascular management of pancreatitis related pseudoaneurysms. A single center experience. Gastroint Int 2014;3:46-53.

7. Laganà $\mathrm{D}$, Carrafiello $\mathrm{G}$, Mangini $\mathrm{M}$, et al. Multimodal approach to endovascular treatment of visceral artery aneurysms and pseudoaneurysms. Eur J Radiol 2006;59:104-11. 